Conference Paper

\title{
Design and development of cost-effective PCB prototyping machine
}

Atta-ul-Mustafa, Shakoor, S., Shoaib, S., Ahmed, W. and Aleem, H.

This is a paper presented at the IEEE Conf. of Young Researchers in Electrical and Electronic Engineering, Moscow, 26-29 Jan. 2021.

Copyright of the author(s). Reproduced here with their permission and the permission of the conference organisers.

\section{Recommended citation:}

Atta-ul-Mustafa, Shakoor, S., Shoaib, S., Ahmed, W. and Aleem, H. (2021), 'Design and development of cost-effective PCB prototyping machine,' in Proc. IEEE Conf. of Young Researchers in Electrical and Electronic Engineering, Moscow, 26-29 Jan. 2021, pp. 2006-2009, doi: 


\section{Design and Development of Cost-effective PCB Prototyping Machine}

\author{
Atta-ul-Mustafa, Haroon Aleem, Subja Shakoor, \\ Waqas Ahmed \\ Department of Electrical Engineering \\ HITEC University \\ Taxila, Pakistan
}

\author{
Sultan Shoaib \\ Faculty of Art, Science and Technology \\ Wrexham Glyndwr University \\ Wrexham, UK
}

\begin{abstract}
The demand for CNC machines in educational institutions and industries is rapidly increasing. This paper presents an affordable model of a $\mathrm{CNC}$ machine that can engrave the circuit's layout on copper plates. Our emphasis on designing this machine is to model a low cost and robust model of CNC machine for PCB prototyping. This CNC machine is a combination of electro-mechanical design and control circuitry. Because of its user-friendly design, it provides great ease in engraving operation. First, a layout is designed by using a standard PCB designing software. Then the finalized layout is converted into G-code. This code contains axis information which is translated by the firmware of the microcontroller. According to the axis information, the controller drives the mechanical assembly with the accuracy of micrometers. The result shows better performance on various circuits and proved to be a costeffective tool for academia and industry.
\end{abstract}

Keywords-computer numeric control machine, printed circuit board, $P C B$ prototyping machine, Arduino controller, G-code

\section{INTRODUCTION}

PCBs are an essential element of electronic industries; conventional methods of $\mathrm{PCB}$ designing are time-consuming and is a hectic process. Commercially available PCB machines are too much expensive and industry can afford this product but an individual student can't buy these products so, the main goal of this paper is to design a low cost and student-friendly product as the demands of efficiency and accuracy are increasing, we want machines to perform our daily tasks, so we can avoid human errors. PCBs are required for industrial and domestic use. Computer Numeric Controlled machine uses computer logic Gcode to control the movement of hardware parts of the machine. The electronic part controls the amount of current passing and the motion of the machine.

Due to low cost and user-friendly handling, the efficiency of the CNC increased. With this feature, this machine can be used in institutions and even in repairing electronic cards to create PCB's by drawing circuit layouts on them. The CNC machine can perform the followings tasks:

- Engraving circuit layout on PCB.

- Engraving any type of shape or symbol on a wooden/plastic piece.

Nowadays CNC is using widely because of its precision and accuracy. Tools motion in 3 axes can be precisely controlled using CNC. The software language to control the movement of the axis is usually G-code. It is commonly used in manufacturing for machining metal and plastic parts. The same concept is used to design PCB board, as PCB designing demands accuracy and precision. To avoid the shorting of the circuit, the $\mathrm{CNC}$ machine can control the motion of the axis precisely by controlling the stepping of the stepper motor. As all functions will be performed using numeric control so they can be repeated in the same manner over and over again with $92 \%$ accuracy.

The rest of the paper is distributed as Section II describes all existing methodologies, frameworks, and research carried out related to our nature of the subject. Section III discusses the working mechanism of the CNC machine. Section IV presents the control techniques required for $\mathrm{PCB}$ layout and also explains how the G-code is translated into a language that controls the moments of the axis to make a precise PCB layout. Section V elaborates on the machine assembling and its working process. Finally, conclusion is drawn in Section VI.

\section{LITERATURE REVIEW}

Balasubramanyam and Prasanthi [1] proposed that the machine can be used for multitasking it can create PCB design by controlling four motors. Madekar et al. [2] explained how by using Arduino we can cut down our product's price. Hidayatullah et al. [3] describe the controlling part of the CNC PCB milling machine. Martheyn et al. [4] tell about the controlling of motors using $\mathrm{CNC}$ machine language (G Code). Prince et al. [5] describe how we can achieve a low-cost CNC machine by using different spare parts. Ginting et al. [6] proposed to design complex 3-axis CNC with the microcontroller and spindle drill can be an effective solution. Daadoo and Daraghmi [7] describe how we can design a lowcost $\mathrm{CNC}$ and how we can perform a control printed circuit.

Jalindar et al. [8] explain how to perform automatic drilling of the CNC machine in which soft wares are best suited to generate the G-code file. Gadhe et al. [9] discussed the design of a PCB milling and drilling machine, where the layout is automatically found out from an image of the circuit in EAGLE software. Chapman [10] in his book tells the controlling of the motors for better performance. Rashid [11] in his book tells the basic concept needed to control the spindle and DC motor. 


\section{SYSTEM ARCHITECTURE}

PCB prototyping machine allows users to design the circuit of their own choice by using any standard PCB designing software. Microcontrollers such as Arduino was used to interface the stepper motor by using standard G-code commands. Before The G-codes executed by milling machine they are first converted to machine-specific directives with the help of the software that resides on the on-board computer which intern operates the milling machine. The software sends instructions to the controllers which control the motion of the stepper motors and move the motor in the specified $\mathrm{x}, \mathrm{y}$, and $\mathrm{z}$ directions. Fig. 1 depicts the complete system architecture.

The complete system consists of three parts which includes software, electronics, and mechanical design. In the software part, the software is used to interface the stepper motor movements up to the required direction. The electronic and mechanical parts are mounted for the movements in the $\mathrm{x}, \mathrm{y}$, and z-axis.

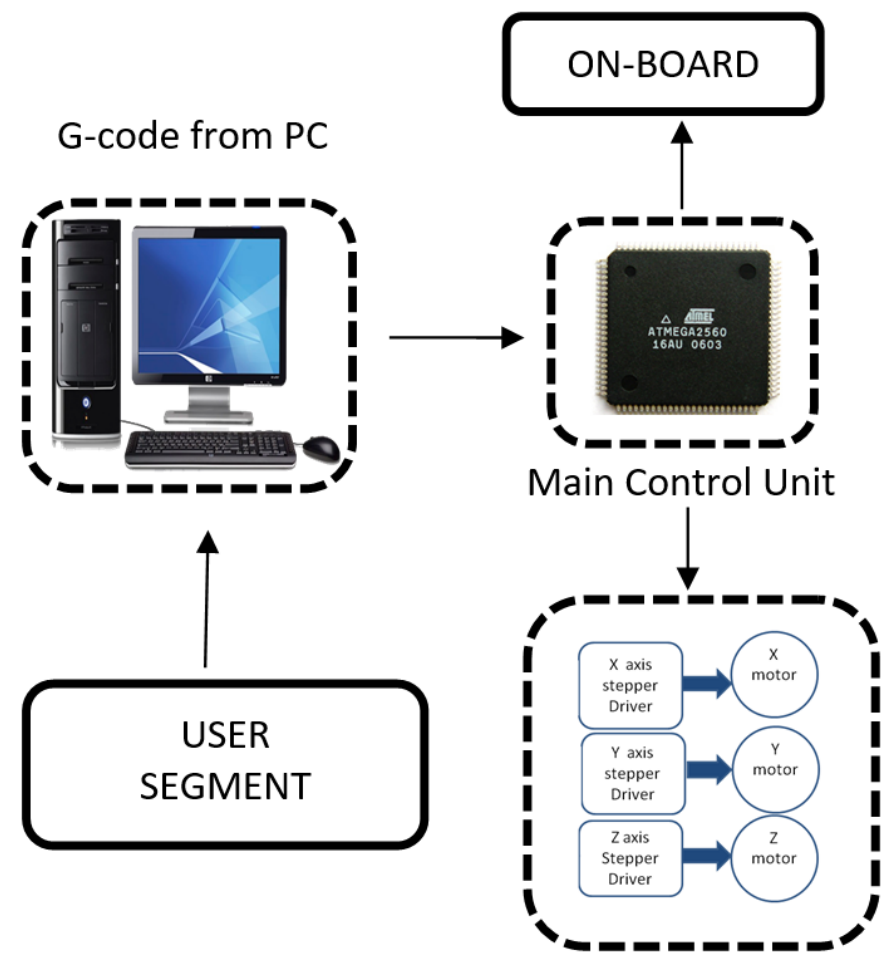

Fig. 1. Block diagram of the complete system.

\section{Simulation Results}

The Main Control Unit (MCU) comprises of controller like Arduino Nano, which perform similar functions of Arduino mega2560 but is in smaller size. It is based on ATmega328 based controller board with 0.73 " $x 1.70$ " dimensions. The working and operations of MCU can be further divided into two parts.

\section{A. Stepper Motor Controlling}

There are two basic types of stepper motor depending on the polarity i.e., Unipolar, Bipolar. In this paper bipolar stepper motor has been used to move the mechanical plates of the CNC machine linear. The bipolar stepper motor has four wires which are further distinguished according to their colour. Different stepping size can perform using a bipolar stepper motor, max it can provide 1/32 step size using A4988 diver IC. To provide a voltage source to the stepper buck converter IC (LM2576) has been used. Buck converter steps down the DC voltage. The power supply output voltage is 24 which is directly given to the spindle DC motor and the same voltages are further step down using buck converter IC. Fig. 2 shows the complete IC interface with stepper motor.

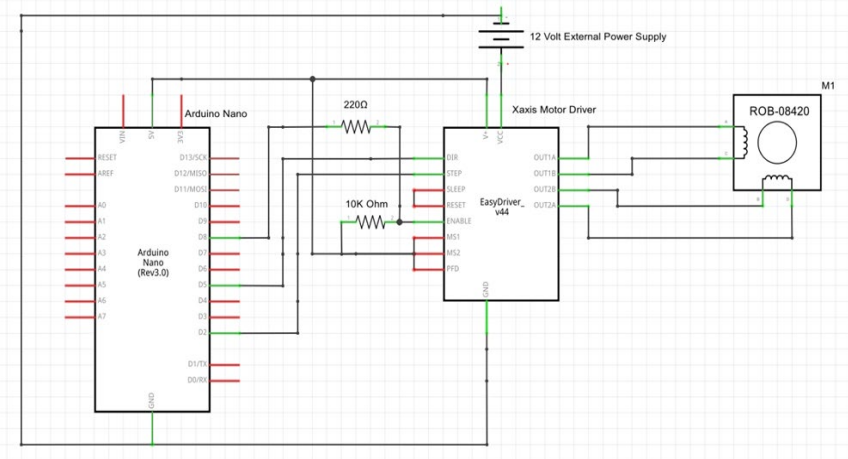

Fig. 2. A4998 driver IC interface with $\mathrm{x}$ axis stepper motor.

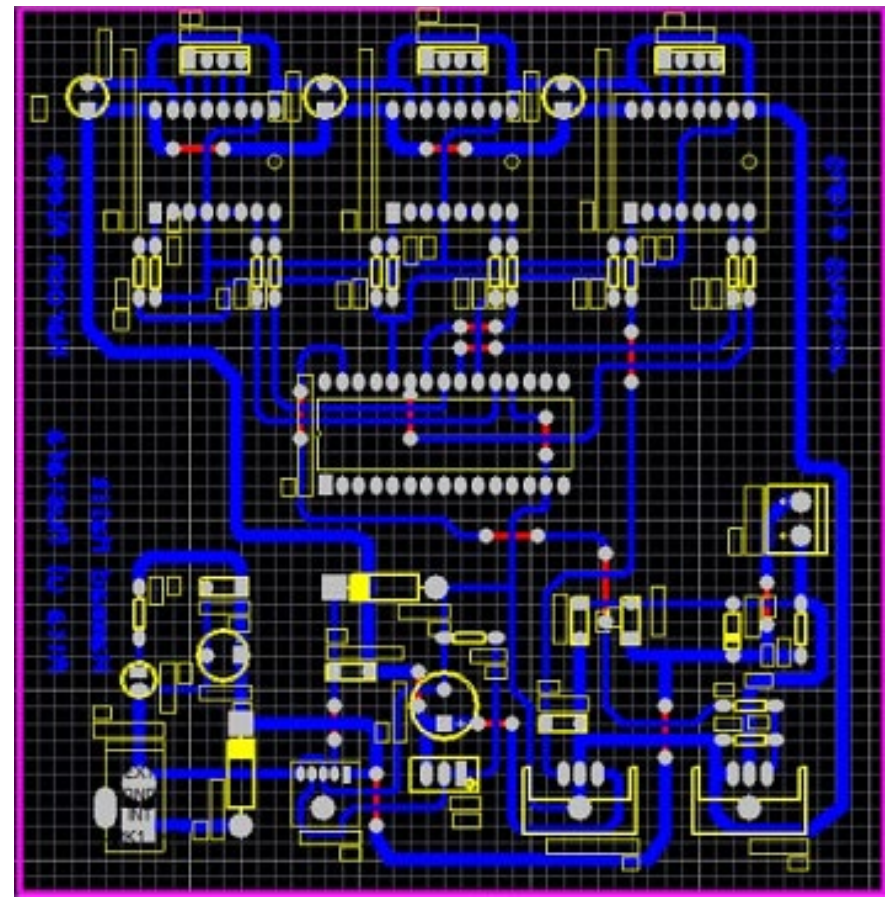

Fig. 3. Control unit of the machine.

To convert the $24 \mathrm{~V}$ source voltage into $12 \mathrm{~V}$, the buck converter is designed. For this purpose, we designed a standalone power supply which is powered up by $24 \mathrm{~V} \mathrm{DC}$. The selection of components was made to keeping in mind the required output voltage. The Buck regulator regulates a voltage from $1.2 \mathrm{~V}$ to $50 \mathrm{~V}$ as shown in Fig. 3. The current rating of the design spindle circuit is $1.3 \mathrm{~A}$. Whereas the motor driver circuit draws up to $1 \mathrm{~A}$ current for its operation. This sums up to a requirement of at least $1.2 \mathrm{~A}$ and at least $12 \mathrm{~V}$. 
The circuit used for the regulator has an adjustable IC (LM2576) which converts the DC supply of 55V into required variable voltages. The variable resistor is used to adjust the exact $12 \mathrm{~V}$ at the output for the spindle motor circuit operation.

\section{B. Control of CNC Machine}

Once the control of the stepper motor is done, the next step is to use the motor to draw the circuit on PCB. The layout of circuit drawing can be obtained using any simulation software. The picture of circuit design has been converted into machine language (G-code) using Inkscape. G-code consists of the information of axis motion and vector form. Then this information is translated to stepper motor using Arduino Nano. Grbl 0.9 controller is given this G-code and finally, it draws the circuit on PCB according to the information given by the Gcode.

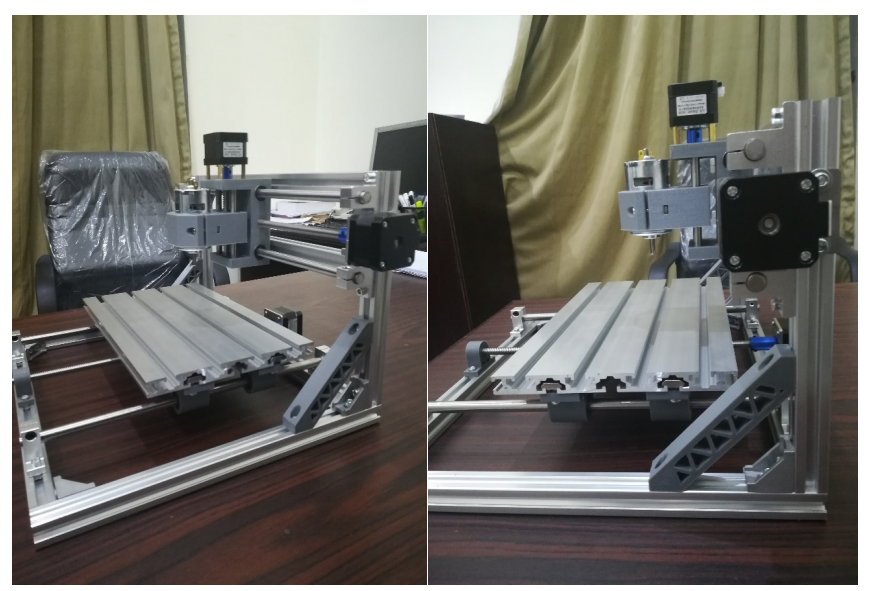

Fig. 4. CNC Machine.

\section{DESIGN AND IMPLEMENTATION}

PCBs are part of every electronic gadget and become an unavoidable need for industries and domestic. Before $\mathrm{CNC}$, the conventional method of PCB designing was time-consuming, as it required etching, the printing of circuits drawing on PCB by a heat gun, so the probability of human error was high. The slightest error can damage the circuit. Computer Numeric Controlled machine or CNC machine is a machine that uses computer logic to control the movement of hardware parts of machine.

This machine consists of mechanical parts and electronic parts which control the machine together they are used to perform the function of the CNC machine, CNC controller has a Grblv0.9 software which decodes the G-code and performs the required functions of the machine. With the rapid advancement in technology, CNC found vast applications for commercial and industrial purposes. These machines are used in industries as lathes, millings, punch presses, laser cutters, and press brakes et cetera.

The main idea is to design a CNC machine that will draw circuit layouts on PCB and will help in the creation of a perfect PCB. Due to numeric controlling PCB can be designed accurately and precisely. The low-cost mechanical parts and a simple controller design makes this machine an economical machine for PCB designing. The main objective in creation of this machine is to develop an automatic mini-CNC machine for PCB drawing. Design and implement the system for three-axis movement and control. To reduce the cost of the machine and increases flexibility.

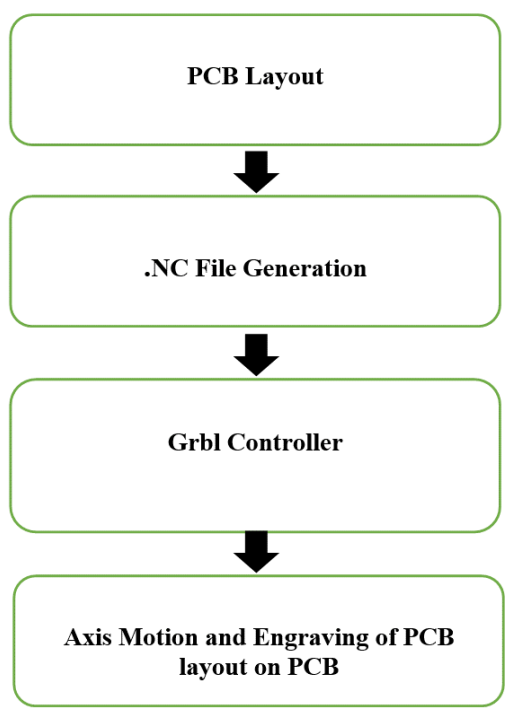

Fig. 5. Effect of modified ground structure on the performance.

The mainframe of the PCB prototype machine is consisting of aluminium and plastic parts. The aluminium body has a workbench and aluminium rods which are mounted together with the help of a lead screw. The side and bottom frame are joined by 2 plastic supports. This frame contains 3 stepper motors and $1 \mathrm{dc}$ spindle motor. These motors perform 3 types of motions: $\mathrm{X}$-axis, $\mathrm{Y}$-axis, $\mathrm{Z}$-axis, $\mathrm{C}$ axis. While its circular motion is including the 4th type i.e., $\mathrm{C}$ axis. These motors rotate in 360 degrees. According to these movements, the machine can perform these types of motions:

- $\quad$ Rapid Motion (Also Called Positioning)

- Straight Line Motion

- Circular Motion

Rapid and straight-line motions are performed in only one direction while circular motion is the movement in 2 axis movements. Each stepper motor defines 1 dimension of movements. The layer of PCB engraving is performed by the combined motion of these motors.

Once the axis is set, click on the open file and select the file PCB design. It will open in Grbl, the pen will appear and will start moving according to the axis information in the file and the design will start printing on PCB. When the file is uploaded and the axis is set to zero position then we check the spindle motor rotation by clicking on the screen spindle icon. We can increase the spindle rotation by moving the icon bar and the rotation of the spindle is controlled.

\section{CONTROL UNIT}

To control the CNC machine, a Control Unit has been used based, which sends the user instructions to the stepper motors which are given by G-code. We have used Inkscape for converting the PCB layout file into a machine language G-code file. After converting that file, we send that file to a Grbl 
controller which will further control the moment of the axis of the machine. After sending the commands the machine reads the G-code and draw the PCB layout on the PCB provided.

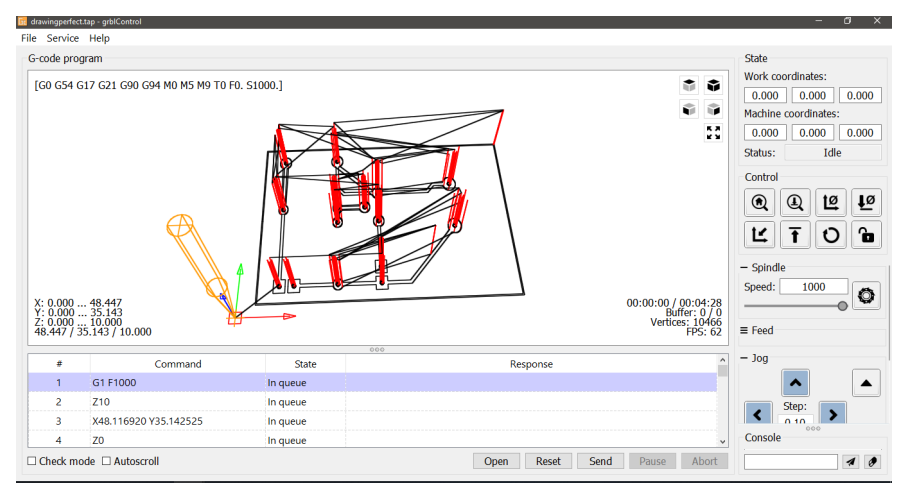

Fig. 6. Grbl v 0.9 Control Unit.

Now user can open the desired circuit from any given PCB layout software and generate its .nc file and open that in Grblv0.9, the interface for Grblv0.9 control unit can be seen in Fig. 6, the main step for this process are:

1. The axis is adjusted to the zero position and the cursor moves towards the zero-axis point and the machine adjusted the position accordingly.

2. The spindle motor starts rotation as soon as we click the Send icon on the Grbl screen.

3. After the procession complete the spindle starts rotating and the machine with the receiving command takes the usage of 3 stepper motors. It takes some time to complete the desired circuit to be complete according to the G-code given.

4. The circuit is then completed by the machine in the required time and the spindle moves back to the initial point where it starts.

5. From initializing the Grbl controller to the required circuit design, the picture completely explained the operation of the CNC milling machine.

\section{CONCLUSION}

This paper depicts the idea of PCB designing using a CNC machine. It is designed to facilitate the institution who cannot buy an expensive CNC machine for their students to make their project on PCB's. This CNC is user friendly and can easily draw circuit layout on PCB with instructions of the user. The combination of Arduino with NEMA (stepper motors) made it possible to control the $\mathrm{CNC}$ machine using $\mathrm{PC}$ and create efficient circuit layouts on PCB's. Arduino Nano was used for controlling the movement of stepper motors. In this way, the user who wants to make a circuit layout do not have to repeat the old conventional method of creating circuit layout on PCB's, he/she just has to draw the circuit layout on any circuit simulating software, generate the required file for the $\mathrm{CNC}$ machine and $\mathrm{CNC}$ will automatically create that circuit layout on PCB as instructed by the user. Thus, in a fast and effective way, the circuit layouts can be produced.

\section{REFERENCES}

[1] N. Balasubramanyam, and G. Prasanthi, "Design and fabrication of an automatic PC-based drilling machine," HCTL Open International Journal of Technology Innovations and Research, vol. 7, pp. 1-13, Jan. 2014.

[2] K.J. Madekar, K.R. Nanaware, P.R. Phadtare, and V.S. Mane, "Automatic mini CNC machine for PCB drawing and drilling," International Research Journal of Engineering and Technology, vol. 3, no. 2, pp. 11061110, Feb. 2016.

[3] M. Hidayatullah, F.I. Hariadi, and A. Sasongko, "Development of interface and coordination module of FPGA-based controller for CNC PCB milling and drilling machine," in Proc. Int. Symp. on Electronics and Smart Devices, Yogyakarta, Indonesia, 17-19 Oct. 2017, pp. 112-117.

[4] J.M. Berbesi, K. Saumeth, and F. Pinilla, "Parallel control firmware for CNC milling machine based in Arduino," in Proc. 12th Int. Microsystems, Packaging, Assembly and Circuits Technology Conference, Taipei, Taiwan, 25-27 Oct. 2017, pp. 319-322.

[5] M.K.K. Prince, M.-Al-M. Ansary, and A.S. Mondol, "Implementation of a low-cost CNC plotter using spare parts," International Journal of Engineering Trends and Technology, vol. 43, no. 6, pp. 333-339, Jan. 2017.

[6] R. Ginting, S. Hadiyoso, and S .Aulia, "Implementation 3-axis CNC router for small scale industry," International Journal of Applied Engineering Research, vol. 12, no. 17, pp. 6553-6558, 2017.

[7] M. Daadoo, and Y.-A. Daraghmi, "Design and implementation of low cost computer numerical control-printed circuit," International Journal of Engineering and Innovative Technology, vol. 5, no. 10, pp. 63-67, April 2016

[8] J.P. Jalindar, K.A. Baburao, C.P. Mahadeo, and T. Chavan, “Automated PCB drilling machine using DIP," International Journal for Scientific Research and Development,| vol. 4, no. 2, pp. 1184-1185, Feb. 2016.

[9] P. Gadhe, V. Jangir, and Wasim-Ul-Haq, "Design and implementation of PCB using CNC," International Research Journal of Engineering and Technology, vol. 4, no. 2, pp. 1721-1725, Feb. 2017.

[10] S.J. Chapman, Electric Machinery Fundamentals, 4th ed., New York: McGraw-Hill, 2005.

[11] M.H. Rashid, Power Electronics: Devices, Circuits, and Applications, 4th ed., Harlow: Pearson, 2013. 